\title{
Doppler Effect Plug-in in Music Production \& Engineering
}

\author{
Yoemun Yun \\ Dept. of Applied Music, Chungwoon University \\ San 29, Namjang-ri, Hongseong, Chungnam, 350-701, South Korea \\ hippie740@chungwoon.ac.kr
}

\begin{abstract}
Sound travels in acoustics. There are numerous audio effects and sound plug-ins in the digital music industry based on the principles of acoustics. In addition, sound is minute fluctuations in pressure. These fluctuations are rapid and are cqused by pressure and velocity of the air. Pressure pushes air molecules which move, and therefore have velocity. Human hearing is capable of responding to fluctuations within the range of 20 fluctuations per second to 20,000 fluctuations per second. Standing waves are generated wherever waves are reflected towards oncoming waves. They are generated when two loudspeakers produce exactly the same sound.

The waves pass through each other but the interference nodes and antinodes seem motionless. The Doppler Effect is observed as a phenomenon in which the pitch of a sound drops as the sound source passes by the observer. The direction and relative speed of the source and observer are important.

This paper describes the concept of Dopplen Effect plug-ins and explains how they work and how they can be utilized(in music production.
\end{abstract}

Keywords: Compression, Rarefaction, Equal Loudness Contour, Doppler Effect, Sound Interference, Standing Waves

\section{Introduction}

A turning fork is an acoustic instrument which produces a pure tone, only one frequency. The rate of pressure fluquations in a pure tone is constant. In this concept, it can be thought that a push in one direction yields a compression, which can be thought of as a slight overpressure, and a push in the opposite direction yields a rarefaction.

Figure 1 démonstrates how compressions and rarefactions travel away from a sound source at the speed of sound. Compressions and rarefactions move in all directions rather equally, esuiting in ever expanding spheres.

The wave under the tuning fork and air is a graphical representation. The peaks above equilibrium correspond to compressions and the valleys below equilibrium correspond to rarefactions.

Wavelength is the distance between consecutive identical portions of sound, such as one peak to the next. Frequency is the number of fluctuations per second, and the speed of sound is expressed in feet per second ( $\mathrm{ft} / \mathrm{s}$ ) and is typically $1130 \mathrm{ft} / \mathrm{s}$, but it can affect by temperature and air pressure. 


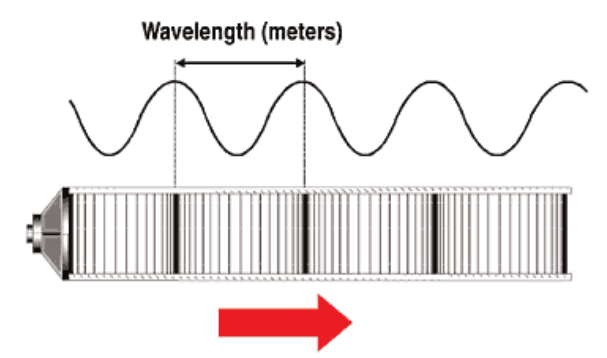

Figure 1. Sound Compression and Rarefaction

\section{Physics of Sound}

\subsection{Equal Loudness Contour and Constructive/Destructive Sound}

Each equal loudness contour indicates the levels of tones adross the range of frequencies which have the same perceived loudness. A $20 \mathrm{~Hz}$ tone would have to be reproduced at a much higher level than a tone at $1000 \mathrm{~Hz}$ in order to sound as loud.

The contours flatten as the pitches get higher. The loudness button on a stereo is meant to compensate for the reduced sensitivity to low frequencies when histening at low levels. Most loudness buttons are only bass boosts. A trueloudness buttongradually reduces bass boost as the overall level is increased.

Human hearing is most sensitive from $2 \mathrm{kHz}$ io $5 \mathrm{kHz}$. This means that the clear human speech which comes primarily from consonant sounds is concentrated within this range of frequencies.

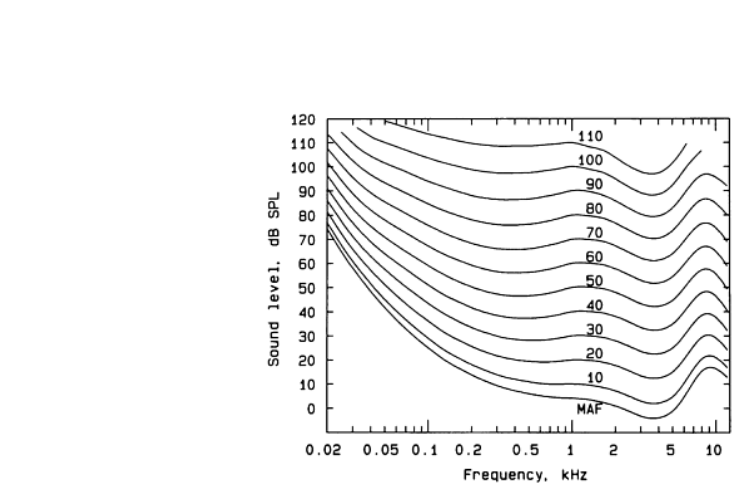

Figure 2. Equal Loudness Contours

Wayes are able to pass through each other and emerge with their original identities. This is called superposition. As portions of the waves occupy the same position, the relative amplitudes are combined. Constructive interference is results in increased amplitude and destructive interference is in decreased amplitude. A vibrating element has both potential energy and kinetic energy. Energy is radiated from the element as sound energy and heat energy. Potential energy corresponds to pressure. When the element is slowest, most of the energy is stored as potential energy. Kinetic energy corresponds to velocity. When the elements are moving at its greatest velocity, the energy is primarily kinetic energy. 


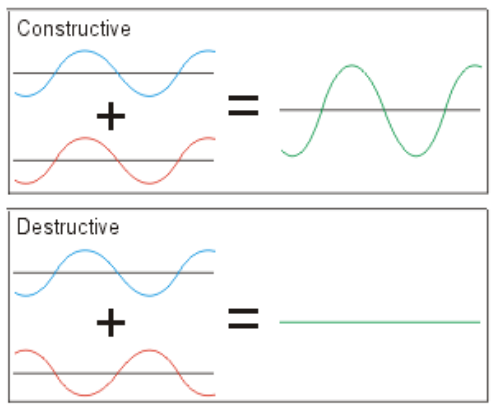

Figure 3. Constructive and Destructive Sound

\subsection{Sound Interference and Standing Waves}

Interference means that similar waves coming from two or more separated sources may either aid or oppose each other. The interference is construetive or destructive according to whether the waves arrive in or out of phase. Two waves or slightly different frequency will go repeatedly in and out of phase with each other to produce beats [1].

Standing waves are generated wherever waves are reflected towards oncoming waves. They are generated when two loudspeakers produce exactly the same sound. The waves pass through each other, but the interference nodes and antinodes seem motionless. The result is called a standing wave, because the nodes and antinodes appear to stand still. There are a great number of standing waves in nost real rooms. The enormous number tends to obscure an individual standing wave. Having two ears serves to smear identification of discrete nodes and antinodes.

When we begin to consider standing waves, some distinct associations come to mind; things such as discrete reflectors, low frequencies, and single microphones. First, discrete reflectors are a reflective surface which is far from other surfaces allows individual standing waves to remain unobscured and problematic.

Due to the nature of low frequency waves, there are many fewer low frequency nodes to obscure each other than high frequency modes nodes. Moreover, the standing wavelengths are much longer and the amplitudes are generally greater, revealing the nodes and antinodes more clearly. In the case of single microphones, increasing the number of microphones consequentially increases the number of nodes received, obscuring individual standing waves.

There are always peaks near the boundaries of rooms. The position of these depends on whether the node has been created between the walls or between the floor and ceiling. The frequencies (f) at which the strongest nodes will occur is given by the equation below [2],

$$
\begin{gathered}
\mathbf{f}=(\mathbf{c} / \mathbf{2}) *(\mathbf{n} / \mathbf{d}) \\
\mathrm{f}=\text { frequency } \\
\mathrm{c}=\text { the speed of sound } \\
\mathrm{d}=\text { dimension } \\
\mathrm{n}=\text { the number of the mode }
\end{gathered}
$$

\subsection{Physics of Doppler Effect}

The Doppler Effect is observed as a phenomenon in which the pitch of a sound drops as the sound source passes by the observer. The direction and relative speed of the source and receiver are important. If the source and receiver move toward each other, the perceived 
frequency increases. If they move away from each other, the perceived frequency decreases. If neither the sources nor the receivers are moving, there is no Doppler effect and no apparent change in frequency.

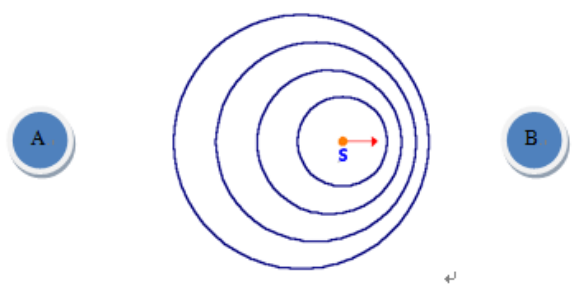

\section{Figure 4. Doppler Effect in Sound Physics}

In Figure 4, wave crests emitted by a source (S) travel in all directions. The outermost wave was emitted when the source was at the origin of the arrow; a sixth crest is now being crested. Observer A receives crests crowded closer ogether than if the source were stationary; for observer B they are spread further apart [1].

There is no Doppler effect if the source and the receiver are moving in the same direction at the same speed. A receiver located in the ppposite direction from the source's motion would encounter compressions and rarefactions less frequently and the apparent frequency would decrease.

The apparent frequency due to the Doppler Effect can be calculated from the equation below.

$$
\begin{aligned}
& f^{\prime}=\mathbf{f s} *(\mathbf{C}+\mathbf{C r} / \mathbf{C}-\mathbf{C s}) \\
& f(f)=\text { apparent frequency } \\
& \mathrm{C}=\text { speed of sound }
\end{aligned}
$$

C) - spleed of the receiver toward sound source $\Omega$

The equation is stated in terms of the speed of the receiver towards the source and the speed of the source towards the receiver. If the receiver or the observer is moving away from each other, the speeds will be negative values.

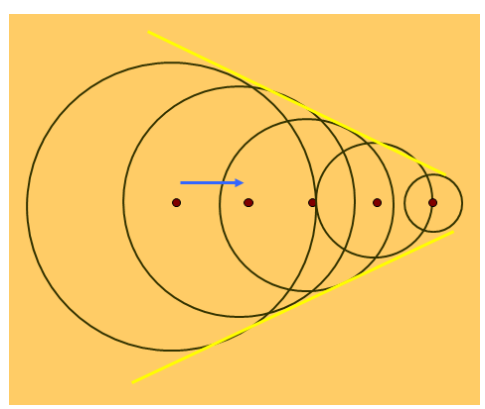

Figure 5. Doppler Effect in Case of Observer Moves 
If the observer is moving toward the sound the effect is similar, but the wave crests have a different relative spacing.

There is a shift for both the pulse out and reflected signal. $f^{\prime}=f(1+u / v)(u=$ observer movers at seed). A moving source can exceed the speed of sound. The sound waves interfere on a front at an angle to the motion [3].

\subsection{Digital Doppler Effect Plug-in}

Digital Doppler Effect simulator plug-ins have various functions. I have chosen to demonstrate the use of the Doppler Effect within music production with the Waves Doppler plug-in;

The engineer can adjust the point at which the effect is at its maximum by adjusting the center node located along the curved line. The nodes located on the left and right of the curved line do not affect the center time.

If audio content having fewer channels than can be provided by a target system is available, the target audio system cannot take full advantage of it. Therefore, in order to utilize such audio content, it is necessary to use an upmixing method that converts mono or stereo audio formats into a multi-channel audio format suitable for such a system [4].

However, the speed of sound image or panning can affect the centre time. To maximize the realness of the effect, there are several parameters, such as, gain (volume of the sound with distance), pan (left and right), pitch (pitch shift), air damp (moves sound away from the listener), reverb (reflections from the stereoor surroundings), and reverb time, which must be adjusted appropriately according to the situation.

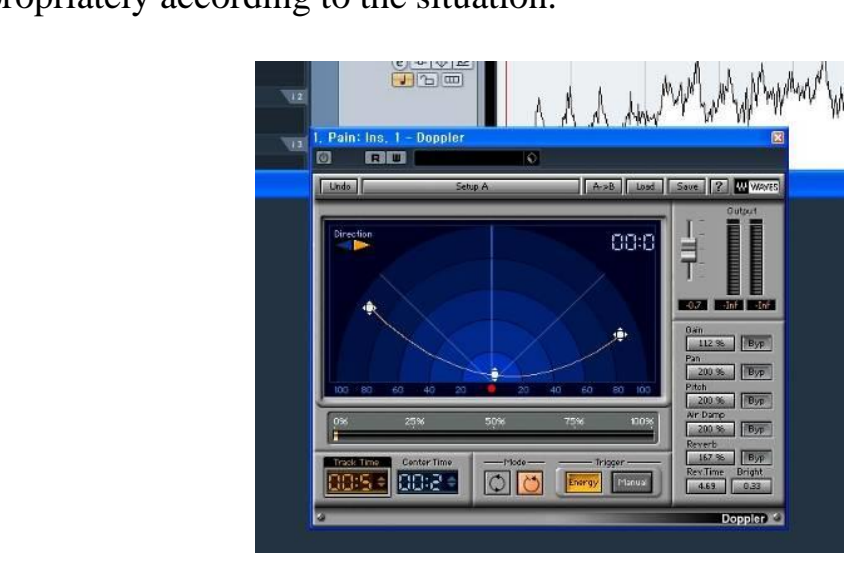

Figure 6. Digital Doppler Effect Plug-in

If users test the programmable type it will then be easy to understand the adjustments that must be made in a preset machine. Both types have stereo outputs and may have either mono or stereo inputs, depending on the model's preferences [5].

In order to make the effect as real as possible, the start and end point of the effect, curve of the track, center time, and tone color controls of the Doppler plug-in must be given careful attention. It is recommended that pitch change, gain, air damping, and other controls are set after first setting these five important parameters, which require critical listening and time in order to produce realistic results. 


\section{Conclusion}

As technology developed, there are numerous audio effects and sound plug-ins in the digital music industry based on the principles of acoustics.

The Doppler Effect is also from the physics sound. Music producers and engineers can use the effect for films such as train or airplane passed by. Doppler Effect also can be used to achieve a chorusing effect by combining the outputs of sounds. In that case, this effect is needed combination of reverb, right pitches, and so on.

\section{References}

[1] D. E. Hall, "Musical Acoustics", $3^{\text {rd }}$ Edition, Books/Cole (2001).

[2] F. Rumsey and T. McCormick, "Sound and Recording", Fourth Edition, Focal Press (2001).

[3] www.niu.edu/ mfortner/PHYS211/.../P211_07f

[4] C. J. Chun, Y. G. Kim, J. Y. Yang and H. K. Kim, "Real-Time Convetsion of Stereo Audio to 5.1 Channel Audio for Providing Realistic Sounds", International Journal of Signal Processing. Indage Processing and Pattern Recognition, vol. 2, no. 4, (2009).

[5] Y. Yun, "Audio Analysis \& Creating Reverberation Plug-ins In Digital/Audio Engineering", International Journal of Multimedia and Ubiquitous Engineering, vol. 8, no. 6, (2013), pp.201-208 http:// dx.doi.org/10.14257/ijmue.
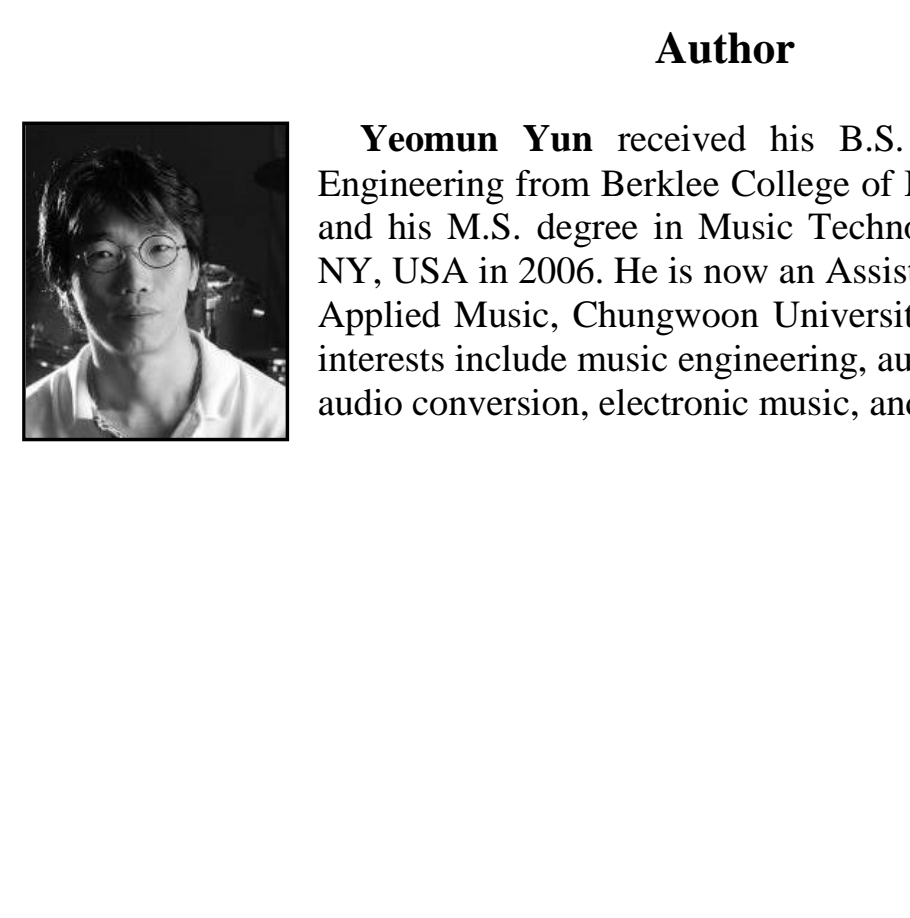

Yeomun Yun received his B.S. degree in Music Production \& Engineering from Berklee College of Music, Boston, MA, USA in 2004, and his M.S. degree in Music Technology from New York University, NY, USAyin 2006. He is now an Assistant Professor in the Department of Applied Music, Changyoon University, Hongsung, Korea. His research interests include music engineering, audio sound design, analog-to-digital audio conversion, electronic music, and computer music composition. 